The Geneva Papers on Risk and Insurance, 21 (No. 79, April 1996), 224-239

\title{
The Ban of a Single Pesticide
}

\author{
by Arndt Rölike*
}

\section{Introduction}

Chemical pesticides are widely used in agriculture in order to protect the crop from several pests or to increase their storability. As has been shown, for example in the US, the use of pesticides to farmers is highly beneficial. It has been estimated in 1978 that the chemical control costs are $\$ 2.2$ billion with a gross return on those costs of $\$ 8.7$ billion (Pimental (1978)). However, it is also well known that pesticides cause damage to the environment. Their use leads to a contamination of the soil, different water bodies like lakes or groundwater, as well as the air. Even the drinking water in modern industrial societies is threatened by contamination from pesticides (Nowotny/Chesters (1981)).

One widely used instrument to protect the environment from a contamination by pesticides is the ban of them. In 199378 countries used this kind of intervention. ${ }^{1}$ However, what is used is the ban of a single, highly toxic pesticide or a very small group of pesticides. Not considered is the ban of all pesticides. This would have disastrous effects on the production of agricultural products. Therefore it is understandable that although the instrument of bans is widely employed, the variety of existing and used pesticides is still enormous. Right now roughly 1000 different pesticides are registered in the Federal Republic of Germany alone although many have been already banned (Ahlheim (1989)). Among those which have been banned are - just to cite a few examples - Dieldrin, Aldrin or Paraquat. ${ }^{2}$

Legal foundation for these product bans in Germany is the law on plant protection, in German called Pflanzenschutzmittelgesetz (PSMG). The german PSMG offers basically two ways for the regulating agency to ban a pesticide. One is to ban the application of a

*University of Hamburg. The paper was presented at the 6 th Joint Conference between the EALE and the Geneva Association. It greatly benefited from a comment by Robin Mason. Furthermore, I owe Georg von Wangenheim, Mark Thomas and an anonymous referee thanks for some helpful discussions. The usual disclaimer applies.

I Compare Hamburger Abendblatt 24.06.1993.

${ }^{2}$ Compare the German PflanzenschutzmittelVO. 
registered pesticide according to cap 7 PSMG. The other is to reject the reregistration of a pesticide after the registration period of 10 years maximum has expired. This is due to cap 16 PSMG. Banning a pesticide according to cap 7 PSMG requires a retraction of the former registration. Since the legal requirements for retraction are much stricter than those for rejection the regulating agency usually takes the latter way (Micklitz (1991)). The regulating agency in Germany is the Biologische Bundesanstalt (BBA). Since the BBA usually bans a pesticide by rejecting reregistration it has to apply the principles for registration. Those principles are formulated in cap 15 PSMG. According to this chapter a pesticide should not be registered if it has negative side effects on the environment which are not "acceptable» on the basis of scientific knowledge. The supreme court of Germany (BVerwG) in its decision on the reregistration of the pesticide Paraquat clarified what has to be taken into consideration with regard to the legal term «acceptable». ${ }^{3}$

According to this decision the court states that on the one hand the negative aspects of damage caused by the product have to be taken into consideration. On the other hand the positive aspects of a pesticide have to be considered. Those positive aspects are determined by the use of the pesticide for agricultural production, and its replaceability. Therefore, the easier it is to replace a pesticide with other pesticides the more the decision should be in favor of a ban.

The BBA drives the argument of the supreme court concerning the replaceability even further by stating that given a pesticide is fully replaceable and that there are no other negative aspects for the agricultural production connected with the ban, the pesticide should be banned without any further investigation. In particular, according to the BBA there is no need for any further comparison of different pesticides, and a comparison between different pesticides also would not accord with the law (Brasse/Bode/Rothert (1990)).

Regarding replaceability of a pesticide as a pure positive aspect, as well as banning without any further investigation in the case of complete substitutability, builds on the same assumption. This assumption is that all other pesticides which are potential substitutes are not harming the environment at all. However, as important as replaceability is - a recent estimation states that substitutes are available for roughly $70 \%$ - the assumption that those substitutes would not be harmful is wrong (Zilberman et. al. (1991)). Instead, it can be said that it is within the nature of pesticides to harm the environment up to a certain extent (Lersner (1982) and Sergerson (1990)). Therefore when looking at a situation where replaceability is given, the harmful effects of the substitutes have to be taken into consideration.

The aim of the article is to highlight some aspects connected with the problem of substitutability on the one hand, and the toxicity of the substitutes on the other hand. In order to do that, a simple model is constructed in section two focusing on these aspects. In section three the ban of a pesticide is discussed within this framework. There the consequences of the supreme court decision are presented. In section four the consequences of the BBA statement are analysed. The results derived in section four are put into a broader context in section five and section six. Thereby, a general problem using the instrument of

${ }^{3}$ BVerwG decision dated from 10th of November 1988 in: 9 Natur und Recht (1989), 385-387. 
banning single toxic goods is developed. Furthermore, a comparison of the pesticide ban to a uniform tax on all pesticides is persued. In section seven the results are summarized.

\section{The model}

In order to keep the analysis as simple as possible a variation of the model developed by Weitzman in 1974 is used (Weitzman (1974)). Thus a benefit function in pesticides is confronted with an environmental damage function. The benefit function is a Taylor Series approximation of the second degree in pesticides added by a term presenting a simple measure for substitutability. ${ }^{4}$ Denoting the quantities of the two pesticides analysed as $q_{a}$ and $q_{b}$ respectively, the benefit function can be written as follows:

(1) $B=b_{0}\left(q_{a}+q_{b}\right)-\frac{1}{2} b_{1}\left(q_{a}+q_{b}\right)^{2}+b_{2} q_{a} q_{b}$

whereby $b_{0}, b_{1}$ and $b_{2}$ are constant parameters. It is assumed that $b_{0}$ and $b_{1}$ are greater than zero. The marginal benefit functions are:

(2) $\frac{d B}{d q_{a}}=b_{0}-b_{1} q_{a}-\left(b_{1}-b_{2}\right) q_{b}$

(3) $\frac{d B}{d q_{b}}=b_{0}-b_{1} q_{b}-\left(b_{1}-b_{2}\right) q_{a}$

With regard to $b_{2}$ a couple of comments are necessary. Note first that $b_{2}$ is a measure for substitutability. Given that $b_{2}$ is equal to zero the two pesticides are perfect substitutes. The benefit is only dependent on the sum of both pesticides. The farmer does not derive any value from having both of them. Given $b_{2}$ is equal to $b_{1}$ the marginal benefit function of each pesticide is independent of the amount of the other pesticide. They are not substitutable at all. Given $b_{2}$ is greater than $b_{1}$ the pesticides could be regarded as complements since in that case an increase in the quantity of one pesticide implies an increase in the marginal benefit of the other pesticide. Therefore, we restrict the analysis on values of $b_{2}$ which are within the range of zero and $b_{1}$ inclusive. Within this range one can see that the lower $b_{2}$, the higher is the dependence of the marginal benefit function of one pesticide on the quantity of the other pesticide and therefore, the higher is the substitutability of the two pesticides.

However $b_{2}$ also has a second meaning which is, at least within this framework, the positive aspect of a low substitutability. This is the valuation of product differentiation.

${ }^{4}$ The general Taylor Series approximation of the second degree in two variables is as follows:

$B=a_{1} q_{a}+a_{2} q b+a_{3} q_{a}^{2}+a_{4} q_{b}^{2}+a_{5} q_{a} q_{b}$

Hence, the following specifications are made:

$a_{1}=a_{2}=b_{0} ; a_{3}=a_{4}=-\frac{1}{2} b_{1} ; a_{5}=b_{2}-b_{1}$

Those specification are made for technical convenience. Note first that the used benefit function has all usually assumed properties like a positive but decreasing marginal benefit up to a specific amount of pesticides or a negative rate of substitution which is decreasing with increasing quantities. Note second that price repercussions of the regulation are neither captured by the general nor captured by the specified benefit function. This is a general drawback of models of this kind which do not explicitly model underlying market mechanisms. For a model taking price repercussions into consideration see Rölike (1994). 
Therefore, given a specific amount of both pesticides the higher $b_{2}$ the higher the benefit of the farmers.

The other component of the model is the environmental damage function. Also here a Taylor series approximation of second degree is used. It is assumed that damage increases at an increasing rate in environmental degradation, denoted with $\mathbf{E}$. Furthermore it is assumed that environmental degradation is a linear function of the quantities of the two pesticides. In order to express different degrees of toxicity of the two pesticides the quantities are weighted with toxicity parameters, denoted by $\tau_{\mathrm{a}}$ and $\tau_{\mathrm{b}}$ respectively. Therefore, the environmental damage function can be written as follows:

(4) $D=d_{0} E+d_{1} E^{2}$

whereby $E$ is equal to:

(5) $E=\tau_{a} q_{a}+\tau_{b} q_{b}$

Again, $\mathrm{d}_{0}, \mathrm{~d}_{1}, \tau_{\mathrm{a}}$ and $\tau_{\mathrm{b}}$ are constant parameters greater than zero.

Note, that expressing toxicity by only one parameter is certainly a strong simplification of the different aspects involved. However, in reality sometimes different toxicities are expressed by one parameter. Parameters of this kind actually used are, for example, LD 50 values. LD 50 values are a measure for how much quantity of a single substance is needed in order to kill $50 \%$ of the organisms analysed (Säle/Knauf (1991)).

Welfare is defined as the difference between benefit and environmental damages.

Thus, the model is fully specified and the analysis of a product ban can be undertaken in the subsequent sections.

\section{Banning one pesticide}

In this section we are going to analyse what the effects of the ban of one pesticide are, given that substitutes are available. Furthermore, we are going to analyse what implication this has on the regulation decision that the supreme court regards substitutability exclusively as a factor which is in favor of a ban.

In order to do that we assume in this section that the regulating agency has full information and that there is no uncertainty.

Given the situation before intervention both pesticides will be used in such an amount that their marginal benefit equals zero. Hence, the quantities are determined by the following two conditions:

(6) $b_{0}-b_{1} q_{a}-\left(b_{1}-b_{2}\right) q_{b}=0$

(7) $b_{0}-b_{1} q_{b}-\left(b_{1}-b_{2}\right) q_{a}=0$

From this follows:

(8) $q_{a}=q_{b}=\frac{b_{0}}{2 b_{1}-b_{2}}$ 
Then, the sum of both pesticides denoted with $\mathrm{Q}$ is equal to:

(9) $Q=\frac{2 b_{0}}{2 b_{1}-b_{2}}$

Note, that these quantities increase in $b_{2}$. This is a result of $b_{2}$ being a measure for $a$ positive evaluation of product differentiation. The more product differentiation is valued, the higher is the benefit deriven from the pesticides and the more they are used. Plugging the quantities of pesticide $a$ and $b$ into the benefit and the damage function and after rearranging terms we get:

(10) $B=\frac{b_{0}^{2}}{2 b_{1}-b_{2}}$

and

(11) $D=d_{0}\left(\tau_{a}+\tau_{b}\right) \frac{b_{0}}{2 b_{1}-b_{2}}+d_{1}\left(\tau_{a}+\tau_{b}\right)^{2}\left(\frac{b_{0}}{2 b_{1}-b_{2}}\right)^{2}$

This situation is obviously not efficient since environmental damage is not taken into account and therefore the aggregate quantity of pesticides is, in comparison to the social optimum, too high. Furthermore the more toxic pesticide which is without any loss of generality assumed to be good $b$, is in comparison to good a used too much.

Obviously, the efficient solution in this framework could be gained by two Pigou taxes equal to the marginal damage of the pesticides in the social optimum. However this is not persued in political and legal reality, at least in Germany. The reason for this objection might be the considerably high administration costs involved with this instrument. Considering the number of different pesticides, up to one thousand different Pigou taxes would have to be created.

Instead, the instrument of banning one of the pesticides is used. Since we want to model a rational ban in a situation of certainty, we will consider a ban of the relatively more toxic pesticide b. Good b can certainly be considered as more toxic as long as the following condition holds:

(12) $\tau_{a}<\tau_{b}$

Given good $\mathrm{b}$ is banned and abstracting from any enforcement problems - which is reasonable with regard to a ban (Bohm/Russel (1985)) - the quantity values after the ban are defined by the following conditions:

(13) $b_{0}-b_{1} q_{a}=0$

(14) $q_{b}=0$

Note that after the ban pesticide $a$ is still allocated suboptimally since the marginal benefit of good a is still equal to zero and not equal to its marginal damage.

Rearranging the first condition leads to a quantity of good a after the ban of:

(15) $q_{a}^{v}=\frac{\mathrm{b}_{\mathrm{o}}}{\mathrm{b}_{1}}$

whereby the $v$ denotes values after the ban. 
Plugging the new quantity of pesticide a into the benefit and the damage function and after rearranging terms yields:

(16) $B^{\mathrm{v}}=\frac{b_{0}^{2}}{2 b_{1}}$

and

(17) $D^{\mathrm{v}}=d_{0} \tau_{a} \frac{b_{0}}{b_{1}}+d_{1} \tau_{a}^{2}\left(\frac{b_{0}}{b_{1}}\right)^{2}$

By considering if the ban of good $b$ is welfare enhancing or not, we have to compare the benefit before and after the ban with the damages before and after the ban. As long as the damage reduction is higher than the benefit reduction, the pesticide ban can be considered as welfare enhancing.

Denoting the differences with $\Delta$ one gets for the benefit reduction:

(18) $\Delta B=B-B^{v}=\frac{b_{0}^{2} b_{2}}{2\left(2 b_{1}-b_{2}\right) b_{1}}>0$

As presumed by the supreme court, the benefit reduction is increasing in $b_{2}$, i.e. a lower substitutability implies a higher cost of the pesticide ban, since the difference in benefit before and after the ban is increasing in $b_{2}$.

(19) $\frac{\partial \Delta B}{\partial b_{2}}=\frac{b_{0}^{2}}{\left(2 b_{1}-b_{2}\right)}>0$

The damage reduction is defined by:

(20) $\Delta D=D-D^{v}$

Using the conditions stated above as well as rearranging terms yields:

(21) $\Delta D=d_{0}\left(\tau_{a}+\tau_{b}\right) \frac{b_{0}}{2 b_{1}-b_{2}}+d_{1}\left(\tau_{a}+\tau_{b}\right)^{2}\left(\frac{b_{0}}{2 b_{1}-b_{2}}\right)^{2}-d_{0} \tau_{a} \frac{b_{0}}{b_{1}}-d_{1} \tau_{a}^{2}\left(\frac{b_{0}}{b_{1}}\right)^{2}$

This term can be better interpreted by noting that the difference of the aggregate quantity of pesticides before and after the ban is equal to:

(22)

$$
\Delta Q=Q-Q^{v}=\frac{b_{0} b_{2}}{\left(2 b_{1}-b_{2}\right) b_{1}}
$$

and the difference in average toxicity is equal to:

(23) $\Delta \bar{\tau}=\bar{\tau}-\tau_{a}=\frac{1}{2} \tau_{a}+\frac{1}{2} \tau_{b}-\tau_{a}=\frac{1}{2} \tau_{b}-\frac{1}{2} \tau_{a}$

Using the last three conditions and rearranging terms the damage reduction can be rewritten as:

(24) $\Delta D=d_{0} \Delta \bar{\tau} Q+d_{1}(\Delta \bar{\tau} Q)^{2}+d_{0} \tau_{a} \Delta Q+d_{1}\left(\tau_{a} \Delta Q\right)^{2}+\varepsilon$

whereby $\varepsilon$ is equal to :

(25) $\varepsilon=d_{1} \tau_{a}\left(\tau_{b}-\tau_{a}\right) Q^{2}+2 d_{1} \tau_{a}^{2} Q^{\nu} \Delta Q>0$ 
Therefore, the effect of banning the more toxic pesticide on environmental damage can be decomposed essentially into two effects:

The first effect is indicated by the decrease in average toxicity represented by the term $\Delta \bar{\tau}$ given the aggregate pesticide quantity is constant. The second effect is a decrease in the total amount of pesticides used given the toxicity of the remaining pesticide. This effect is represented by the terms including $\Delta Q$. The term $\varepsilon$ is simply representing the fact that both effects are stronger with a quadratic damage function than merely squaring the linear effect. Since both effects are reducing environmental damage one can in this case state that the ban of the more toxic pesticide clearly decreases environmental damage.

However, taking the derivative of the damage reduction for $b_{2}$ one can see as well that this is positive. It is equal to:

$$
\begin{aligned}
& \frac{\partial \Delta D}{\partial b_{2}}=d_{0} \Delta \bar{\tau} \frac{\partial Q}{\partial b_{2}}+d_{1}(\Delta \bar{\tau})^{2} 2 Q \frac{\partial Q}{\partial b_{2}}+d_{0} \tau_{a} \frac{\partial \Delta Q}{\partial b_{2}}+d_{1} \tau_{a}^{2} 2 \Delta Q \frac{\partial \Delta Q}{\partial b_{2}}+d_{1} \tau_{a}\left(\tau_{b}-\tau_{a}\right) 2 Q \frac{\partial Q}{\partial b_{2}}+2 d_{1} \tau_{a} Q^{v} \frac{\partial \Delta Q}{\partial b_{2}} \\
& \quad \text { whereby }
\end{aligned}
$$

$$
\frac{\partial Q}{\partial b_{2}}=\frac{\delta \Delta Q}{\delta b_{2}}=\frac{2 b_{0}}{\left(2 b_{1}-b_{2}\right)}>0
$$

Hence, the higher substitutability implies a lower reduction of pesticides on the one hand and on the other hand a lower positive effect of the decrease in toxicity. The latter effect occurs since the quantity of pesticides before the ban is lower, as well given a higher $\mathbf{b}_{2}$. Both effects imply that a higher substitutablity of the pesticides causes not only lower costs of the ban but also a lower benefit of the ban. By taking only the former effect of a high substitutablity into account, the principles of the supreme court clearly lead to a bias in favour of the ban.

Whether the ban is welfare increasing or not depends on if the following condition holds:

(28) $\Delta D>\Delta B$

This clearly depends on the specific parameter constellation.

\section{Banning randomly}

In the last section it was proved that considering substitutability exclusively as an argument in favor of the pesticide ban - as the supreme court suggested - creates a bias towards banning too many pesticides. The BBA derived the argument of substitutability even further by stating that given perfect substitutability the pesticide will be banned without any further investigation. The underlying idea is that given perfect substitutes are available the ban of a product creates no costs. This is true within the framework of this model where no price changes are considered. ${ }^{5}$ In order to see that, assume a situation of perfect substitutablity, i.e. $b_{2}$ is equal to zero. From this follows:

\footnotetext{
5 When price changes are taken into consideration this argument is not true. For a discussion of this problem see Rölike (1994).
} 
(29) $\Delta B=\frac{b_{2} b_{0}^{2}}{\left(2 b_{1}-b_{2}\right) b_{1}}=0$

Therefore, the question of whether the pesticide ban is welfare enhancing or not is entirely dependent of the change in environmental damage. If the more toxic pesticide is banned, environmental damage decreases and the ban is unambiguously welfare enhancing. However, following the statement of the BBA no comparison of the products is undertaken. So refering to the situation of the model it is sufficient for the BBA to observe that $\mathrm{q}_{\mathrm{a}}$ and $\mathrm{q}_{\mathrm{b}}$ are perfect substitutes in order to ban one of the products. Hence, which of the two pesticides is banned depends only on the question of which registration period ends first. If product b's registration period ends first the BBA observes the availability of a perfect substitute and bans it. If product a`s registration period ends first the BBA would ban this pesticide since the same observation would be made and no further investigation would be undertaken. Taking the BBA statement serious implies that the chances for product a to be banned are as high as the chances for product $b$ to be banned and they equal $50 \%$.

Therefore, expected change in environmental damage is equal to:

(30) $E[\Delta D]=\frac{1}{2} \Delta D_{a}+\frac{1}{2} \Delta D_{b}$

whereby $\Delta D_{\mathrm{a}}$ denotes the change of environmental damage in case product a is banned and $\Delta D_{\mathrm{b}}$ in case of product $\mathrm{b}$ is banned. As has been shown in section three $\Delta D_{\mathrm{b}}$ equals:

(31) $\Delta D_{b}=d_{0} \Delta \bar{\tau} Q+d_{1}(\Delta \bar{\tau} Q)^{2}+d_{0} \tau_{a} \Delta Q+d_{1}\left(\tau_{a} \Delta Q\right)^{2}+d_{1} \tau_{a}\left(\tau_{b}-\tau_{a}\right) Q^{2}+2 d_{1} \tau_{a}^{2} Q^{v} \Delta Q$

Since a situation of perfect substitutes is assumed $\Delta Q$ is equal to zero i.e. there is no reduction in aggregate quantity of pesticides. ${ }^{6}$ Therefore, the term for the damage reduction in case of product $b$ is banned reduces to:

$$
\Delta D_{b}=d_{0} \Delta \bar{\tau} Q+d_{1}(\Delta \bar{\tau} Q)^{2}+d_{1} \tau_{a}\left(\tau_{b}-\tau_{a}\right) Q^{2}
$$

Given pesticide $a$ is banned the difference in environmental damage is equal to:

$$
\Delta D_{a}=-d_{0} \Delta \bar{\tau} Q+d_{1}(-\Delta \bar{\tau} Q)^{2}+d_{1} \tau_{b}\left(\tau_{a}-\tau_{b}\right) Q^{2}
$$

Therefore, the expected change in environmental damage is:

$$
E[\Delta D]=-\frac{1}{4} d_{1} Q^{2}\left(\tau_{a}-\tau_{b}\right)^{2} \leqslant 0
$$

Since the term is smaller than zero as long as the pesticides differ in toxicity, this implies that banning randomly increases rather than decreases environmental damage given a situation of perfect substitutability. Therefore taking the statement of the BBA serious, their regulating activity with respect to perfect substitutes would lead to an increase of environmental damage.

\footnotetext{
${ }^{6}$ Compare the equation defining the change in aggregate quantity of pesticides number 22 .
} 
The reason for this result has to be found in increasing marginal damage. Those increasing marginal damage imply that the ban of the less toxic product leads to a higher increase in environmental damage than the ban of the more toxic pesticide implies a reduction in environmental damage.

This explanation also can be demonstrated graphically. Given the quantity of pesticides as it is the case in a situation of perfect substitutability, environmental damages are exclusively dependent on average toxicity. Marginal damage with respect to average toxicity is then:

(35) $\frac{\partial D}{\partial \bar{\tau}}=d_{0} Q+2 d_{1} \bar{\tau} Q^{2}$

This is a linear function in average toxicity. Average toxicity in case of product a has been banned is $\tau_{b}$; where product $b$ has been banned it is $\tau_{a}$ and in case of no intervention it is the average of both. The following graph depicts the situation:

\section{Figure 1}

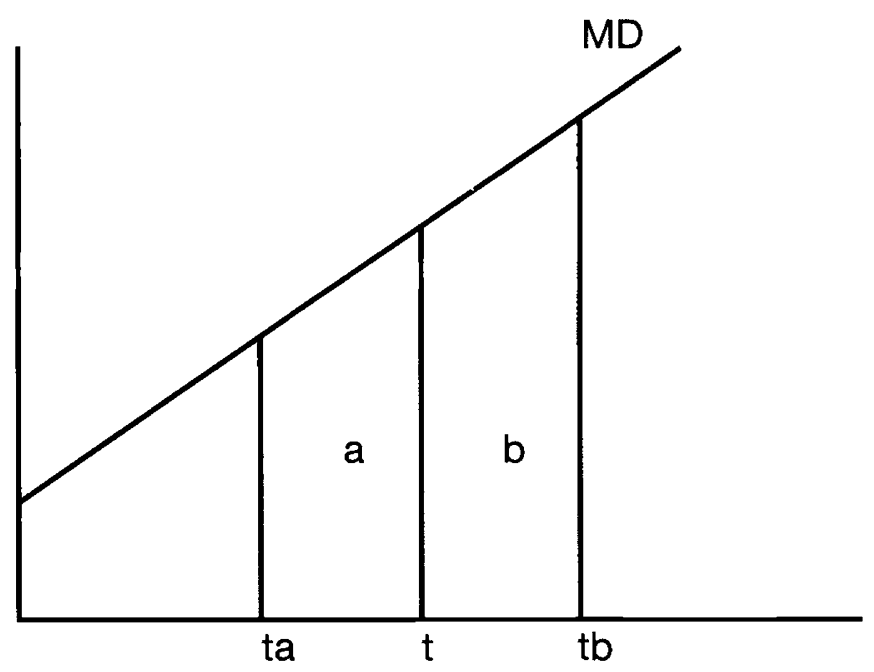

The damage reduction where pesticide $b$ has been banned and the damage increase where pesticide a has been banned are depicted by the area a and $b$ respectively. Since area $b$ is greater than area $a$ the random ban leads to an expected increase in environmental damage.

Therefore, we can summarize this section by stating that in case of perfect substitutability and random picking, as the BBA wrongly suggest, environmental damage increases. The reason for this result is that because of increasing marginal damage, a reduction of damage when the more toxic pesticide is banned is more than offset by the increase in the case of banning the less toxic one. Therefore, as long as the BBA insists on not comparing 
different substitutes with respect to their toxicity, their regulating activity cannot be regarded as useful for protecting the environment.

\section{Lack of knowledge}

So far the BBA has been criticized for not comparing different pesticides with regard to their toxicity. Where no comparison is persued it has been shown that in this case damages increase instead of decrease given the same amount of aggregate pesticide. On the other hand it also has been shown that as the more toxic pesticide is banned, this leads to an improvement in the environmental situation via a decrease in average toxicity and via a decrease in the total amount of pesticides. This observation leads to the question as to whether it would be possible for the regulating agency to detect the more toxic product. Clearly, the answer to this question is highly dependent on the knowledge of the BBA and the knowledge of society generally about environmental damage. The lower this knowledge is, the less one is able to say which absolute toxicity a single pesticide has and the lower also is the possibility to detect a pesticide as a more toxic one in comparison to the other. This is shown by the following argument.

Assume that unknown parameters exist for the toxicities of the two pesticides. Society and especially the BBA try to find out those unknown parameters by conducting experiments, trying to understand the complex system of nature and the disturbance of this system by the pesticides. ${ }^{7}$ Thereby, the BBA could come to some conclusion about the toxicity of the products. Given the BBA would react rationally it would compare the estimated parameters and then ban the product which is supposed to be more toxic. This can be demonstrated graphically as follows:

Figure 2

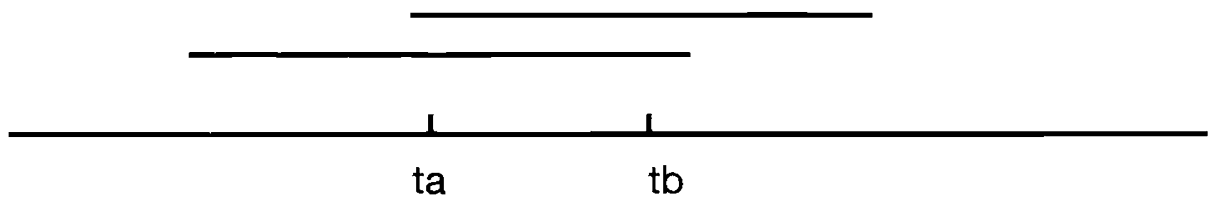

whereby ta and tb are indicating now the unknown true parameters. The lines above the parameters indicate the range of the BBA estimates. The higher the range the less the BBA and society know about the true toxicities. Given equal and independent distribution of the estimates of the BBA around the true parameters in the interval $\mathrm{x}$, then the probability for the BBA to assume pesticide $b$ to be the more toxic product is equal to:

$$
\text { (36) } \operatorname{Prob}\left(\tau_{b}>\tau_{a}\right)=1-\frac{1}{2} \frac{x-\left(t_{b}-t_{a}\right)}{x} \frac{x-\left(t_{b}-t_{a}\right)}{x}=\frac{\left(x-\left(t_{b}-t_{a}\right)\right)^{2}}{x^{2}}
$$

\footnotetext{
${ }^{7}$ On the difficulties which are connected with the estimation of toxicities see Covello/Merkhofer (1987).
} 
At the limit, ie. in case of $x$ approaching infinity, this probability tends to $1 / 2$. This is exactly the situation we have described in the last section. In that section the reason for banning randomly was a result of the incorrect approach taken by the BBA. Since the BBA refuses explicitly to compare toxicities it bans randomly. The reason in this section for banning randomly is the lack of knowledge of society about the true toxicity parameters. Although the given reasons are different the consequences are pretty much the same. In both cases the environmental damage is likely to increase, given the aggregate quantity of pesticides is constant. Therefore, if the BBA were to take the correct approach by systematically comparing the toxicities of the different possible substitutes before banning any of them, the chances of the product ban being a beneficial policy instrument would be lower the lower the knowledge about the complexity of the environmental system is. Given basically nothing or close to nothing is known, the selective ban is even likely to increase environmental damage given the same aggregate quantity of pesticides.

The critical probability denoted by $\mathrm{p}^{\mathrm{c}}$, i.e. the probability of banning the more toxic pesticide in order to ensure an improvement for the environment is defined by the following condition:

$$
E[\Delta D]=\left(1-p^{c}\right) \Delta D_{a}+p^{c} \Delta D_{b} \geqslant 0
$$

Replacing the terms for $\Delta D_{\mathrm{a}}$ and $\Delta D_{\mathrm{b}}$ and rearranging terms for the critical probability gives:

$$
\text { (38) } p^{c} \geqslant \frac{1}{2}+\frac{1}{4} \frac{d_{1} t_{b} Q-d_{1} t_{a} Q}{d_{1} t_{b} Q+d_{0}+d_{1} t_{a} Q}
$$

As can be seen easily within the framework of the model, this critical probability is greater than one half and smaller than three quaters. Hence, as long as the true probability of banning the more toxic good is greater than three quarters, the product ban leads to an improvement in the environmental situation and thereby an increase in welfare given perfect substitutability. However given the true probability is smaller than the indicated critical probability, the status quo (ie. the situation without any regulation) is preferable to a situation of regulation by product bans. Whichever situation is given critically depends on the knowledge society, and in specific the BBA, has about chemical substances and their influence on the environment.

\section{Comparison of the selective product ban with a uniform tax on both pesticides}

So far the pesticide ban was compared with a situation of no regulation, ie. with the status quo. The reason for this approach was the argument that a differentiated Pigou tax might be too costly to implement. However a uniform Pigou tax does not appear to be extremely costly. Therefore, it is reasonable to compare a product ban not only with the status quo but also with other forms of regulation. In this section we consider a uniform Pigou tax on both pesticides.

The starting point of this comparison is the observation made in section three, that a pesticide ban usually also implies some reduction in the aggregate quantity of the pesticides. However this reduction could be more cheaply reached by a uniform tax on pesticides. This is well known and can be easily verified within the framework of the model. 
Whereas the benefit in case of a product ban is equal to:

(39) $B^{v}=\frac{b_{0}^{2}}{2 b_{1}}$

in the case of a uniform tax on both pesticides guaranteeing the same pesticide reduction it is equal to:

(40) $B^{s}=\frac{b_{0}^{2}}{2 b_{1}}+\frac{b_{2} b_{0}^{2}}{4 b_{1}^{2}}$

whereby the s denotes valves after the implementation of the tax.

As long as $b_{2}$ is greater than zero, the benefit in the case of a uniform tax is higher than it is in case of a product ban.

Therefore in order to abstract from this well known argument against a regulation by standards, it is useful to compare the product ban and the uniform tax in a situation of perfect substitutability. Thereby the main feature of the product ban (a reduction in toxicity) can be compared with the main feature of a tax (a reduction in aggregate quantity of pesticides). Furthermore, we want to abstract from difficulties to detect the more toxic product as such. We assume that the BBA detects product $b$ to be the more toxic product with $a$ probability equal to one. Hence, the change in welfare in the case of pesticide $b$ being banned is equal to:

(41) $W^{v}-W=\Delta D_{b}=d_{0} \Delta \bar{\tau} Q+d_{1}(\Delta \bar{\tau} Q)^{2}+d_{1} \tau_{a}\left(\tau_{b}-\tau_{a}\right) Q^{2}$

If an improvement in the situation is persued by a uniform tax, denoted by $s$, the regulating agency maximizes welfare over $\mathbf{s}$ by taking into consideration that the aggregate quantity of pesticides is dependent on s. Hence, the optimal tax rate is determined by the following maximization process:

$$
\begin{aligned}
& \underset{s}{\max }: W(Q) \\
& \text { s.t.: } Q=\frac{b_{0}-s}{b_{1}}
\end{aligned}
$$

Solving this problem implies an optimal tax rate of:

(42) $\mathrm{s}=\frac{d_{0} \bar{\tau} b_{1}+2 b_{0} d_{1} \bar{\tau}^{2}}{b_{1}+2 d_{1} \bar{\tau}^{2}}$

Therefore, the aggregate quantity in case of a uniform tax is equal to:

(43) $Q^{s}=\frac{b_{0}-d_{0} \bar{\tau}}{b_{1}+2 d_{1} \bar{\tau}^{2}}$

Hence, the change in welfare caused by the implementation of a uniform tax is determined by:

$$
\text { (44) } W^{s}-W=\left(b_{0}-d_{0} \bar{\tau}\right)\left(Q^{s}-Q\right)-\left(\frac{1}{2} b_{1}-d_{1} \bar{\tau}^{2}\right)\left(\left(Q^{s}\right)^{2}-Q^{2}\right)
$$


Replacing the pesticide quantities and rearranging terms gives the following change in welfare:

$$
\text { (45) } W^{s}-W=\frac{\left(b_{1} d_{0} \bar{\tau}+2 b_{0} d_{1} \bar{\tau}^{2}\right)^{2}}{2 b_{1}^{2}\left(b_{1}+2 d_{1} \bar{\tau}^{2}\right)}
$$

As can be seen easily, the uniform tax as well as the ban of product b imply an increase in welfare. In order to see which instrument of regulation is preferable, we have to compare the difference between the welfare in the case of a uniform tax and the welfare in the case of pesticide $b$ being banned. This difference is determined by the following equation:

(46) $W^{s}-W-\left(W^{\nu}-W\right)=\frac{\left(b_{1} d_{0} \bar{\tau}+2 b_{0} d_{1} \bar{\tau}^{2}\right)^{2}}{2 b_{1}^{2}\left(b_{1}+2 d_{1} \bar{\tau}^{2}\right)}-d_{0} \frac{b_{0}}{b_{1}}\left(\bar{\tau}-\tau_{a}\right)-d_{1} \frac{b_{0}^{2}}{b_{1}^{2}}\left(\bar{\tau}^{2}-\tau_{a}^{2}\right)$

Rearranging terms gives the following condition:

$$
W^{s}-W^{v}=\frac{2 b_{0} b_{1} d_{0} \bar{\tau}\left(2 d_{1} \bar{\tau} \tau_{a}-b_{1}\right)+2 b_{0}^{2} d_{1} \bar{\tau}^{2}\left(2 d_{1} \tau^{2}{ }_{a}-b_{1}\right)+\gamma}{2 b_{1}^{2}\left(b_{1}+2 d_{1} \bar{\tau}^{2}\right)}
$$

whereby gamma is equal to:

$$
\gamma=b_{1}^{2} d_{0}^{2} \bar{\tau}+2 b_{0} b_{1}^{2} d_{0} \tau_{a}+2 b_{0}^{2} b_{1} d_{1} \tau_{a}^{2}>0
$$

As can be seen the question of the uniform tax being preferable to the product ban is crucially dependent on the relative slopes of the marginal damage function and the marginal benefit function. ${ }^{8}$ This result is similar to that gained by Weitzman in the case of uncertainty. As long as the marginal damage function after the product ban is steeper than the marginal benefit function, the uniform tax is unambiguously preferable to the ban of pesticide $b$. Furthermore the difference is crucially dependent on the toxicity of pesticide a. If its toxicity is equal to zero, the product ban is preferable to the uniform tax. If its toxicity is equal to the toxicity of pesticide $b$ the uniform tax is preferable to the product ban. The economic explanation for this result is straightforward. If pesticide a is highly toxic, a product ban cannot reduce environmental damage whereas a uniform tax can. If product $a$ is not very toxic both instruments can reduce environmental damage. However the steeper the benefit function is, the more costly is a reduction of the aggregate quantity of pesticide. Therefore the greater expense is an improvement in the environmental situation via a tax. On the other hand the steeper the damage function is, the more important it is to decrease aggregate quantity of pesticides and the stronger is the drawback of the product ban.

This result also can be depicted graphically:

\footnotetext{
${ }^{8}$ It is indicated by the term $2 d_{1} \tau_{a}^{2}-b_{1}$.
} 


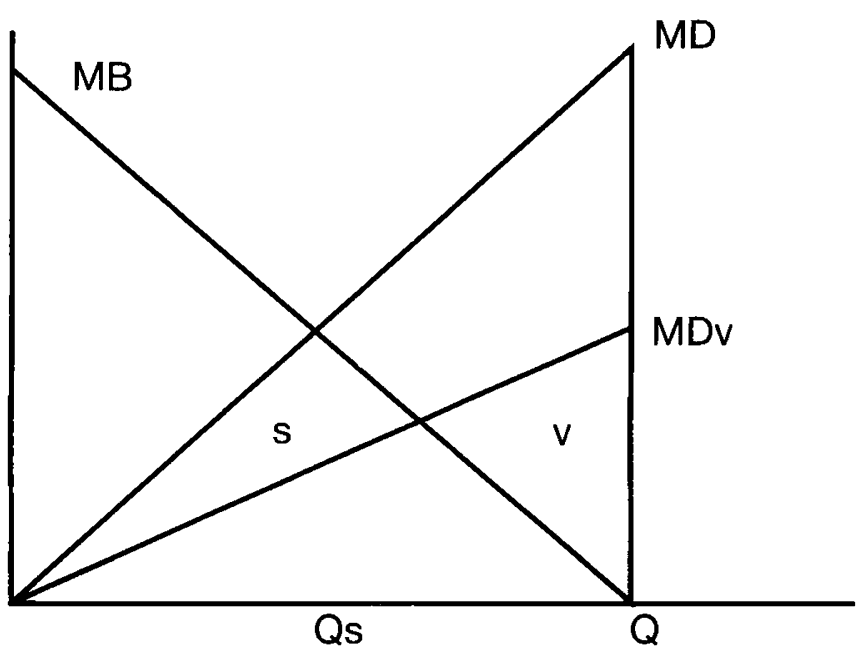

The marginal benefit function, denoted by MB, is depicted together with the marginal damage function before the ban, denoted by MD; after the ban, denoted with MDv. In the case of pesticde $b$ being banned, the aggregate quantity of pesticides is constant and the slope of the marginal damage funtion has decreased as a result of this ban. In the case of a uniform tax, the slope of the marginal damage function is constant whereas the quantity is reduced from $Q$ to Qs. Hence the question of whether the uniform tax is preferable or not crucially depends on whether triangle $v$ is greater or smaller than triangle $s$. In the former case the uniform tax is unambiguously preferable to the product ban. Since the size of both triangles depend on the relative slopes of the marginal damage and the marginal benefit function, the derived result follows immediately.

Therefore this section can be summarized by stating that even if the regulating agency is able to detect with a probability of one the more toxic pesticide, and even if a situation of perfect substitutability is given (a situation where a product ban is costless), this does not imply that this instrument should be used. Instead it should be compared with other instruments. One possible instrument is a uniform tax on all pesticides. As long as the slope of the marginal benefit function is smaller than the slope of the damage function after the ban, it should not be used as a policy instrument in order to protect the environment.

\section{Conclusion}

In the subsequent sections it has been shown that the supreme court and the BBA are not correct in the way they consider substitutability in the case of a pesticide ban. Although substitutability has to be regarded as an extremely important aspect where a ban is applied, this aspect is suitable not only in order to lower the costs of a ban. Instead, it changes the feature of a ban. When considering substitutability, one cannot regard the 
product ban as an extremely restrictive instrument for protecting the environment but as an extremely selective instrument. Being a highly selective instrument, the main feature of the product ban is to compare different substitutes and to select the right one, i.e. to select the most toxic one. However comparing the pesticides seems to be precisely what the BBA has yet to persue. The reason for that might be that the BBA does not know enough in order to make judgements about the relative toxicity of different pesticides. For this kind of judgements not only very precise knowledge about the properties of the different pesticides is needed, but also precise knowledge about the complex system of the environment is important; certain knowledge, for example, that the LD 50 value of a pesticide regarding a specific algae is not enough. One also has to know the implications of this algae on the environment. Furthermore, one has to know how the specific pesticides are used. For example, if 1 miligram of pesticide a is less toxic than one miligram of pesticide $b$ this does not imply that pesticide $a$ is truely less toxic than pesticide $b$ since no statement has been made about the application of the pesticides. It could well be that for production purposes much less of pesticide $b$ is needed than of pesticide $a$. This argument could be driven further towards the agricultural output and the consumption patterns of consumers.

It is possible that the BBA anticipates all those problems and for this reason made the statement about not comparing the toxicities of different pesticides. However if the BBA believes that it is not readily able to compare different pesticides, then a selective instrument such as the product ban should not be implied. Instead, a uniform instrument such as a uniform Pigou tax on all pesticides should be used. This would have the advantage of being a cheaper instrument for protecting the environment. But it would also avoid the damage increase which is likely to occur if product bans are used randomly. And even if the knowledge of the regulating agency is sufficient in order to detect the most toxic pesticide, this also does not imply that this policy instrument is better than other policy instruments. Indeed as a comparison with a uniform tax on all pesticides has shown, this crucially depends also in a situation of certainty on the relative slopes of the marginal damage and the marginal benefit function.

To summarize, the product ban is an extremely common regulatory response which has to be regarded as highly selective but not particularly restrictive. As a result, the main feature of it is to compare different toxicities - a requirement the regulating activity of the BBA in Germany seems not to meet. The ban should not be used where the knowledge for such a selective policy is inadequate. It should furthermore not be persued if the slope of the damage function is very steep in comparison to the slope of the benefit function. 


\section{REFERENCES}

AHLHEIM, K.-H. (1989): Die Umwelt des Menschen, Mannheim ,Wien, Zürich 3. ed.

BOHM, P. and RUSSEL, C. (1985): «Comparative Analysis of Alternative Policy Instruments,» in Handbook of Natural Resource and Energy Economics, Kneese, A. and Sweeney, J. (Ed.), New York, London, Amsterdam : North Holland, 395-461.

BRASSE/BODE/ROTHERT (1990): «Abwägungs- und Entscheidungsprozesse bei der Zulassung von Pflanzenschutzmitteln im Hinblick auf deren Auswirkungen auf die terristische Fauna,» Gesunde Pflanzen 42, 29-33.

COVELLO, V.T. and MERKHOFER, M. (1987): "The Inexact Science of Chemical Hazard Risk Assessment: A Description and Critical Evaluation of Available Methods,» Insuring and Managing Hazardous Risks: From Seveso to Bophal and Beyond, Kleindorfer, P., R. and Kunreuther, H. (Ed.), Berlin : Springer, 229-277.

KELLER, H. (1991): Chemischer Pflanzenschutz in der Landwirtschaft, München : Campus Verlag.

LERSNER, H.v. (1982): «Das dritte Medium,» Natur und Recht 32, 201-205.

MICKLITZ, H.-W. (1991): «Die Zulassungskontrolle von Pflanzenschutzmitteln im Lichte der Paraquat-Entscheidung des Bundesverwaltungsgerichts,» in Bremer Kolloquium über Pflanzenschutz (Tagungsband), Rehbinder, E. (Ed.), Düsseldorf, 146-158.

NOWOTNY, V. and CHESTER, G. (1981): Handbook of Nonpoint Pollution Sources and Mangement; New York, Toronto, Melbourne.

PIMENTEL, D., et al (1978): «Benefits and Costs of Pesticide Use in U.S. Food Production,» BioSciences 28, 772-783.

RÖLIKE, A. (1994): Efficiency Analysis of a Product Ban, unpublished manuscript.

SÄLE, M. and KNAUF, W. (1991): «Planzenschutzmittel und Natruhaushalt - Prüfung, Gefährdungsabschätzung und Risikobeurteilung,» Gesunde Pflanzen 42 (1991), 129-131.

SERGERSON, K. (1990): «Liability for Groundwater Contamination from Pesticides,» Journal of Environmental Economics and Management 19, 227-243.

SHECHTER, M. (1985): «An Atonomy of Groundwater Contamination Episode,» Journal of Environmental Economics and Management 12, 72-88.

WEITZMAN, M.L. (1974): «Prices vs. Quantities» Review of Economic Studies 41, 683-691.

ZILBERMAN/SCHMITZ/CASTERLINE/LICHTENBERG/SIEBERT (1991): «The Economics of Pesticide Use and Regulation,» Science 253, 518-522. 